\title{
铜基材料电催化硝酸盐还原研究进展
}

\author{
汪昌红 ${ }^{1 \dagger}$, 刘正阳 ${ }^{1,2 \dagger}$, 李长明 ${ }^{1,2 *}$, 郭春显 ${ }^{{ }^{*}}$ \\ 1. 苏州科技大学材料科学与工程学院, 苏州 215009 ; \\ 2. 青岛大学生命科学学院前沿交叉研究院, 青岛 266071 \\ $\dagger$ 同等贡献 \\ * 联系人, E-mail: ecmli@swu.edu.cn; cxguo@usts.edu.cn
}

2021-04-05 收稿, 2021-05-19 修回, 2021-06-23 接受, 2021-06-25 网络版发表 国家自然科学基金(21972102)资助

\begin{abstract}
摘要 现代工农业发展迅速, 使得地下水体中的硝酸盐污染越发严重, 已经危害到人类的健康和生态系统的平衡. 电催化硝酸盐还原法是将电化学与催化技术相结合而发展起来的一种新型水处理技术. 该方法通过外加电流使硝 酸盐在阴极处发生电化学还原反应, 进而转化为氮气或有经济价值的氨. 该过程的实现主要取决于阴极电催化材 料. 铜基材料因其固有的硝酸盐还原电催化活性成为人们研究的热点. 目前研究者主要从单质铜、单原子铜、铜 合金、铜基复合材料等 4 个方面进行了探索，但在铜基材料的结构与电催化硝酸盐还原性能之间的构效关系方面 仍然缺乏相关的综述报道. 本文结合最新的研究实例, 总结了铜基材料作为催化剂在电催化硝酸盐还原方面的研 究进展, 论述了硝酸盐电化学还原的机理以及铜基材料结构与电催化硝酸盐还原之间的构效关系, 并进一步讨论 了铜基材料电催化硝酸盐还原的未来发展方向和实际应用所面临的挑战.
\end{abstract}

关键词电催化, 硝酸盐还原, 铜基材料, 构效关系

硝酸盐作为一种非配体形成的氧负离子, 具有很 高的流动性, 且易溶于水 ${ }^{[1,2]}$. 近年来, 随着人类工农业 生产的发展和其他社会活动的增加使得硝酸盐污染越 发严重, 如化石燃料的燃烧、富氮肥料的过量使用以 及污水或废水的排放, 且随着时间的推移而逐渐累积 和广泛分布 ${ }^{[3 \sim 6]}$. 水体中高浓度的硝酸盐会导致水体富 营养化, 降低水的有效氧含量, 导致一些水生生物的死 亡, 破坏水生生态系统 ${ }^{[710]}$. 人体摄人过多硝酸盐会导 致高铁血红蛋白血症(又称“蓝宝宝综合征” ${ }^{[7,11]}$, 主要 是由于硝酸盐被胃肠道微生物还原为亚硝酸盐，从而 把血红蛋白中的亚铁离子氧化成三价铁离子 ${ }^{[12,13]}$. 因 此, 硝酸盐污染防治已成为备受瞩目的全球性问题.

目前为止，去除污水中硝酸盐的方法可分为 3 类: 生物法 ${ }^{[14]} 、$ 物理法 ${ }^{[15]}$ 和化学还原法 ${ }^{[16,17]}$. 生物法主要
是通过微生物体内的酶对其进行还原, 该方法有效、 经济, 关键在于找到合适的菌种 ${ }^{[18]}$. 然而, 生物法面临 的问题是工业污水成分不稳定, 严重影响微生物的还 原活性; 其产生的污泥后处理成本较高, 且耗时长 ${ }^{[19]}$. 物理法去除水中的硝酸盐主要有离子交换法、反渗透 法和电渗析法 ${ }^{[20]}$. 虽然利用物理法去除水中的硝酸盐 具有操作简单、效率高、便于自动化等优点 ${ }^{[21]}$, 但是 离子交换树脂和渗透膜价格较昂贵, 使用寿命有限, 运 行成本过高 ${ }^{[22,23]}$. 化学法是利用化学催化剂将硝酸盐 直接还原为氮气 $\left(\mathrm{N}_{2}\right)$ 或具有经济价值的氨 $\left(\mathrm{NH}_{3}\right)^{[13]}$. 其 主要方法包括光催化还原法、液相还原法和电催化还 原法等. 光催化还原法的原理是利用高能量紫外光照 射反应溶液，产生高还原能力的自由基，进行还原反 应. 此方法具有合成简单、毒性低及成本低等优势, 但 
是存在紫外光吸收范围窄、光能利用率低、溶液透光 度差等问题 ${ }^{[24]}$. 液相催化还原法的基本原理是氧化-还 原反应, 该方法易操作、工艺简单、对设备要求较低, 但是催化效率低、产物选择性差, 不利于后续实验的 进行.

近来, 电催化还原法去除硝酸盐引起研究者的广 泛关注. 其主要通过外加电流在阴极上负载具有特定 催化活性的材料, 达到降解转化硝酸盐的目的 ${ }^{[25 ~ 28]}$. 与 生物法和化学法相比, 其具有以下独特的优势: (1) 电 催化硝酸盐还原法可通过选择特定的电极材料和实验 参数, 高效可控地合成无毒害 $\mathrm{N}_{2}$ 或具有经济价值的 $\mathrm{NH}_{3}$ 等产物. (2) 在电化学反应过程中, 既不引人任何杂质, 也不会对环境产生不利影响, 只需外加一定的电流, 就 可以达到还原硝酸盐的目的. (3) 电催化还原法所需要 的电能可以从可再生的清洁能源中获得, 既丰富了可 再生能源的存储与转换方式, 又在一定程度上降低了 投资成本. (4) 电催化硝酸盐还原技术操作简单, 设备 规模可调，使得将其纳人现有的工艺过程中相对容易， 在去除污水中的硝酸盐方面具有巨大潜力 ${ }^{[29,30]}$. 因此, 发展电催化还原法去除硝酸盐成为未来解决硝酸盐污 染以及资源化的一个有效途径.

目前, 电催化硝酸盐还原技术的发展和应用重点 在于阴极催化剂材料的研究. 良好的阴极催化剂材料 必须具备稳定性好、抗腐蚀性强、导电率高、寿命 长、选择性好等优势 ${ }^{[31]}$. 先前的研究表明, 铅 $(\mathrm{Pb})$ 、镍 $(\mathrm{Ni})$ 、锡 $(\mathrm{Sn})$ 、锌 $(\mathrm{Zn})$ 、铑 $(\mathrm{Rh})$ 、钉 $(\mathrm{Ru})$ 、铱 $(\mathrm{Ir})$ 、钯 $(\mathrm{Pd}) 、$ 铜 $(\mathrm{Cu})$ 、银 $(\mathrm{Ag})$ 和金 $(\mathrm{Au})$ 等催化材料都具有一 定的硝酸盐还原活性 ${ }^{[32 ~ 34]}$. 其中, 铜由于导电性好、析 氢能力弱、催化性能稳定等优势, 引起研究者的广泛 关注. 例如, $\mathrm{Fu}$ 等人 ${ }^{[3]}$ 报道了 $\mathrm{Cu}(111)$ 纳米片电催化硝酸 盐还原合成 $\mathrm{NH}_{3}$ 的速率是普通 $\mathrm{Cu}$ 箔的 400 倍, 是 $\mathrm{Cu}$ 纳米 块的 3.1 倍, 是 $\mathrm{Cu}$ 纳米颗粒的 1.7 倍, 大大提升了电催化 硝酸盐还原的性能. 同时, Zhang等人 ${ }^{[35]}$ 研究发现 $\mathrm{Fe} / \mathrm{Cu}$ 电极电催化硝酸盐去除率高达 $98.6 \%$, 是泡沫铜的 4 倍、纯铁的 1.3 倍, 极大提高了硝酸盐的去除率. 目前, 针对铜基催化剂开展了系列研究 ${ }^{[36,37]}$. 但是, 在铜基材 料的结构与电催化硝酸盐还原性能之间的构效关系方 面还缺乏相关的综述报道.

本文从单质铜、单原子铜、铜合金、铜基复合材 料 4 个方面总结了铜基材料作为电催化硝酸盐还原中 的阴极的最新研究进展, 并且结合大量研究实例论述 了硝酸盐电化学还原的机理和铜基材料结构与电催化
硝酸盐还原之间的构效关系，并进一步讨论了铜基材 料未来发展的方向和实际应用所面临的挑战(图1).

\section{1 电催化硝酸盐还原机理}

目前电催化硝酸盐还原的原理有以下 2 个途径.

（1）首先硝酸根 $\left(\mathrm{NO}_{3}{ }^{-}\right)$在电极表面吸附，随后被还 原为亚硝酸根 $\left(\mathrm{NO}_{2}{ }^{-}\right)$, 这一步是硝酸盐还原速率的限速 步骤 ${ }^{[38-40]}$. 生成的 $\mathrm{NO}_{2}{ }^{-}$在电极表面迅速反应生成吸附 态的一氧化氮(NO), 吸附态的 $\mathrm{NO}$ 从电极表面脱附进人 溶液中, 溶液中的 $\mathrm{NO}$ 与吸附态的 $\mathrm{NO}$ 可以形成弱吸附 的一氧化二氮 $\left(\mathrm{N}_{2} \mathrm{O}\right)$, 进一步被还原为 $\mathrm{N}_{2}{ }^{[41]}$. 具体反应 路径如下:

$\mathrm{NO}_{3}^{-}{ }_{(\mathrm{aq})} \rightleftharpoons \mathrm{NO}_{3}^{-}{ }_{\text {(ads) }}$

$\mathrm{NO}_{3}^{-}$(ads) $+2 \mathrm{H}^{+}+2 \mathrm{e}^{-} \rightarrow \mathrm{NO}_{2}^{-}$(ads) $+\mathrm{H}_{2} \mathrm{O}$

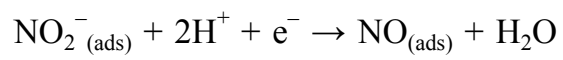

$\mathrm{NO}_{(\text {ads })}+6 \mathrm{H}^{+}+5 \mathrm{e}^{-} \rightarrow \mathrm{NH}_{4}^{+}+\mathrm{H}_{2} \mathrm{O}$

$\mathrm{NO}_{(\text {ads })} \rightarrow \mathrm{NO}_{(\mathrm{aq})}$

$\mathrm{NO}_{(\text {ads })}+\mathrm{NO}_{(\mathrm{aq})}+2 \mathrm{H}^{+}+2 \mathrm{e}^{-} \rightarrow \mathrm{N}_{2} \mathrm{O}_{(\mathrm{ads})}+\mathrm{H}_{2} \mathrm{O}$

$\mathrm{N}_{2} \mathrm{O}_{(\mathrm{ads})}+2 \mathrm{H}^{+}+2 \mathrm{e}^{-} \rightarrow \mathrm{N}_{2}+\mathrm{H}_{2} \mathrm{O}$

(2) 通过质子氢 $(\mathrm{H})$ 介导. 反应开始于水 $\left(\mathrm{H}_{2} \mathrm{O}\right)$ 和 $\mathrm{NO}_{3}{ }^{-}$在电极上的吸附, 并迅速达到动态平衡. 当电极表 面施加恒定电流时，将水还原生成质子 $\mathrm{H}$, 质子 $\mathrm{H}$ 可以 还原吸附的 $\mathrm{NO}_{3}^{-}$, 这一步为限速步骤 ${ }^{[39,42]}$. 质子 $\mathrm{H}$ 还可

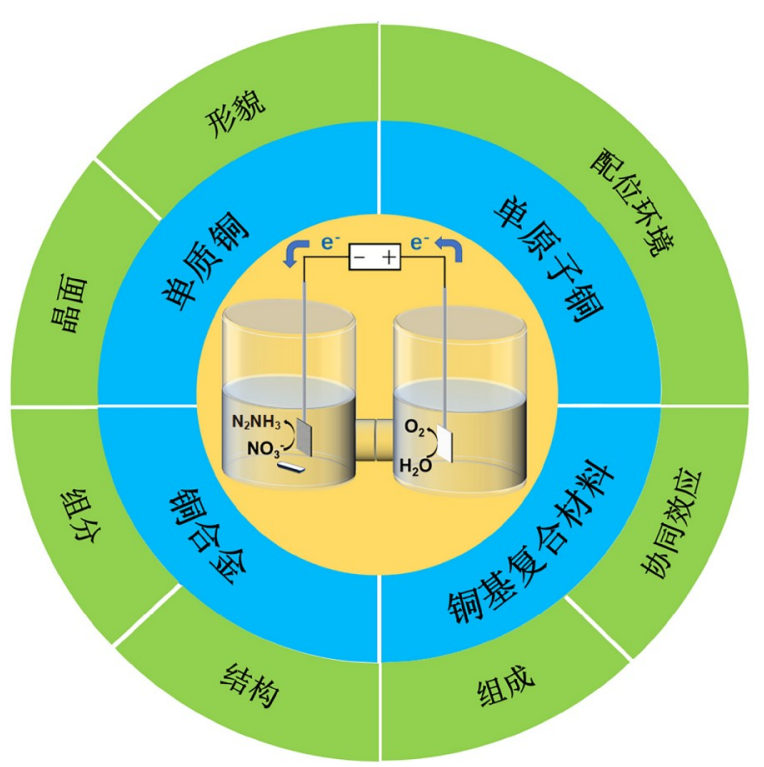

图 1 (网络版彩色)铜基材料电催化硝酸盐还原策略示意图 Figure 1 (Color online) Schematic diagram of electrocatalytic nitrate reduction strategy for copper-based materials 
以还原中间产物 $\mathrm{NO}_{2}{ }^{-}$和 $\mathrm{NO}$, 由于质子 $\mathrm{H}$ 介导的 $\mathrm{N}-\mathrm{H}$ 键 的形成比 $\mathrm{N}-\mathrm{N}$ 键的形成在动力学上更加有利. 因此, 该 过程中主要的产物是 $\mathrm{NH}_{3}{ }^{[43]}$. 反应路径如下:

$$
\begin{aligned}
& \mathrm{NO}_{3}^{-}(\mathrm{aq}) \\
& \mathrm{H}_{2} \mathrm{O}+\mathrm{e}^{-} \rightarrow \mathrm{NO}_{3(\mathrm{ads})}^{-}+\mathrm{OH}^{-} \\
& \mathrm{NO}_{3}^{-}{ }_{(\mathrm{ads})}+2 \mathrm{H}_{(\mathrm{ads})} \rightarrow \mathrm{NO}_{2}^{-}{ }_{(\mathrm{ads})}+\mathrm{H}_{2} \mathrm{O} \\
& \mathrm{NO}_{2}^{-}{ }_{(\mathrm{ads})}+\mathrm{H}_{(\mathrm{ads})} \rightarrow \mathrm{NO}_{(\mathrm{ads})}+\mathrm{OH}^{-} \\
& \mathrm{NO}_{(\mathrm{ads})}+2 \mathrm{H}_{(\mathrm{ads})} \rightarrow \mathrm{N}_{(\mathrm{ads})}+\mathrm{H}_{2} \mathrm{O} \\
& \mathrm{N}_{(\mathrm{ads})}+\mathrm{H}_{(\mathrm{ads})} \rightarrow \mathrm{NH}_{(\mathrm{ads})} \\
& \mathrm{NH}_{(\mathrm{ads})}+\mathrm{H}_{(\mathrm{ads})} \rightarrow \mathrm{NH}_{2(\mathrm{ads})} \\
& \mathrm{NH}_{2(\mathrm{ads})}+\mathrm{H}_{(\mathrm{ads})} \rightarrow \mathrm{NH}_{3(\mathrm{ads})}
\end{aligned}
$$

其中部分 $\mathrm{NH}_{3(\mathrm{ads})}$ 会继续还原生成铵根离子 $\left(\mathrm{NH}_{4}{ }^{+}\right)$:

$\mathrm{NH}_{3(\mathrm{ads})}+\mathrm{H}_{(\mathrm{ads})} \rightleftharpoons \mathrm{NH}_{4}^{+}$(ads)

在电催化硝酸盐还原的整个过程中涉及的反应中 间体以及副产物众多. 因此, 利用精确设计的催化剂来 揭示电催化硝酸盐还原合成 $\mathrm{NH}_{3}$ 或 $\mathrm{N}_{2}$ 的反应动力学和 催化机制是目前电催化硝酸盐还原领域关键的科学问 题，将有利于指导设计具有高选择性和高法拉第效率 的催化剂.

\section{2 铜基材料电极电催化硝酸盐还原}

\section{1 单质铜电催化硝酸盐还原}

研究发现单质铜作为阴极催化剂电催化硝酸盐还 原的活性相对较高, 同时铜自身析氢能力较弱, 因此引 起了研究者广泛的关注. 对铜催化剂的调控主要集中 在其三维形貌结构上：通过减小构筑单元的尺寸来增 大比表面积; 利用表面结构缺陷降低反应能垒; 改变构 筑单元暴露的晶面优化催化性能 ${ }^{[44]}$. 表1总结了不同三 维形貌结构的单质铜催化剂电催化硝酸盐还原的性能.

Reyter等人 ${ }^{[45]}$ 在不同气体条件下利用球磨法研磨
$\mathrm{Cu}$ 粉形成不同结构的 $\mathrm{Cu}$ 纳米颗粒催化剂, 并进行了硝 酸盐还原合成 $\mathrm{NH}_{3}$ 的研究. 其中, 普通铜粉进行球磨处 理后，能显著提高铜电催化硝酸盐还原产 $\mathrm{NH}_{3}$ 的性能. 研究表明, 在空气中研磨的 $\mathrm{Cu}$ 纳米颗粒比在氩气中研 磨的 $\mathrm{Cu}$ 纳米颗粒硝酸盐还原率高 10 倍. 相比于氩气中 研磨的 $\mathrm{Cu}$ 纳米颗粒，在空气中获得的颗粒尺寸更小、 形状不规则、表面存在大量缺陷，都有利于硝酸盐的 吸附和还原产 $\mathrm{NH}_{3} . \mathrm{Fu}$ 等人 ${ }^{[3]}$ 制备了 $\mathrm{Cu}$ 纳米片构型作 为高效产 $\mathrm{NH}_{3}$ 的催化剂. 从透射电子显微镜(TEM)图中 可以看出, $\mathrm{Cu}$ 纳米片呈现规则的三角形结构(图2(a)), 高分辨率TEM(HRTEM) 证实了 $\mathrm{Cu}$ 纳米片主要为 $\mathrm{Cu}$ (111)晶面所对应的铜晶格间距(图2(b))。X射线衍射 (XRD)图显示了高强度的 $\mathrm{Cu}(111)$ 峰, 证明了 $\mathrm{Cu}$ 纳米片 被 $\mathrm{Cu}(111)$ 基准面覆盖. 进一步的电化学测试结果表明, 在相对标准氢电极(RHE) $-0.15 \mathrm{~V}$ 的电位下, $\mathrm{Cu}$ 纳米片 的产氨率为 $390.1 \mu \mathrm{g} \mathrm{mg}_{\mathrm{Cu}}{ }^{-1} \mathrm{~h}^{-1}$, 是 $\mathrm{Cu}$ 管的 400 倍, $\mathrm{Cu}$ 纳 米立方体的 3.1 倍, $\mathrm{Cu}$ 纳米颗粒的 1.7 倍, 法拉第效率高 达 $99.7 \%$. 同时证实 $\mathrm{Cu}(111)$ 面能够有效抑制析氢反应 活性, 而且显著提高电催化硝酸盐产 $\mathrm{NH}_{3}$ 的选择性(图2 (b) (f)). $\mathrm{Wu}$ 等人 ${ }^{[46]}$ 利用 $\mathrm{Cu}$ 纳米带 $(\mathrm{CuNSs})$ 作为催化剂, 其具有比表面积大、界面活性高、电子传递速度快等 优点. $300^{\circ} \mathrm{C}$ 煅烧工艺制备的 CuNSs 呈现出 $0.5 \sim 1 \mu \mathrm{m}$ 的 立方形状; $\mathrm{CuNSs}$ 较薄, 厚度仅为5 10 nm, 纳米片之间 的间隔约为 $50 \sim 150 \mathrm{~nm}$. 此外, CuNSs 是起皱的, 但不重 叠, 可以提供较大的表面积, 促进电化学反应过程的传 质. 而且, 当体系中不加入氯化钠 $(\mathrm{NaCl})$ 、反应 $6 \mathrm{~h}$ 时, 硝酸盐还原率高达 $99.7 \%, \mathrm{NH}_{3}$ 的选择性为 $93.3 \%$; 当体 系中加人 $2 \mathrm{~g} / \mathrm{L} \mathrm{NaCl}$ 、反应 $4 \mathrm{~h}$ 时, $\mathrm{N}_{2}$ 的选择性为 $97.2 \%$. 因此, 在 $\mathrm{Cl}^{-}$的辅助下, $\mathrm{NH}_{4}^{+}$氧化过程启动, 电催化系统 可以有效地将 $\mathrm{NO}_{3}{ }^{-} 、 \mathrm{NO}_{2}{ }^{-}$和 $\mathrm{NH}_{4}{ }^{+}$转化为 $\mathrm{N}_{2}$, 且大大提 高了硝酸盐还原速率. Wang 等人 ${ }^{[47]}$ 报道了一种 $3 \mathrm{D}-\mathrm{Cu}$ 纳米带催化剂作为 $\mathrm{NO}_{3}{ }^{-}$还原的催化剂. 由于其较大的 比表面积, 当外加电位为 $-1.4 \mathrm{~V}(\mathrm{vs} . \mathrm{Ag} / \mathrm{AgCl})$ 时, $3 \mathrm{D}-$

表 1 单质铜电极在硝酸盐还原中的电化学性能总结

Table 1 Summary of electrochemical performance of copper electrodes for nitrate reduction reaction

\begin{tabular}{ccccccc}
\hline 阴极材料 & 阴极面积 $\left(\mathrm{cm}^{2}\right)$ & 电解液 & $\mathrm{NO}_{3}{ }^{-}$转化率 $(\%)$ & 选择性 $(\%)$ & 时间 $(\mathrm{h})$ & 文献 \\
\hline $\mathrm{Cu}$ 纳米颗粒 & 0.785 & $0.1 \mathrm{~mol} / \mathrm{L} \mathrm{NO}_{3}{ }^{-}+1 \mathrm{~mol} / \mathrm{L} \mathrm{NaOH}(75 \mathrm{~mL})$ & $73 \sim 74$ & $97\left(\mathrm{NH}_{4}{ }^{+}\right)$ & 8 & {$[45]$} \\
$\mathrm{Cu}$ 纳米片 & 10 & $140 \mathrm{mg} / \mathrm{L} \mathrm{NO}_{3}{ }^{-}+0.1 \mathrm{~mol} / \mathrm{L} \mathrm{Na}_{2} \mathrm{SO}_{4}$ & 99.7 & $97.2\left(\mathrm{~N}_{2}\right)$ & 6 & {$[46]$} \\
$\mathrm{Cu}$ 纳米带 & 4 & $30 \mathrm{mg} / \mathrm{L} \mathrm{NO}_{3}{ }^{-}+0.5 \mathrm{~mol} / \mathrm{L} \mathrm{Na}_{2} \mathrm{SO}_{4}(50 \mathrm{~mL})$ & 100 & $\left.-^{-}\right)$ & 1 & {$[47]$} \\
$\mathrm{Cu}$ 纳米颗粒 & 75 & $100 \mathrm{mg} / \mathrm{L} \mathrm{NO}_{3}^{-}(200 \mathrm{~mL})$ & 60 & $\left.-{ }^{-a}\right)$ & 1.5 & {$[48]$} \\
\hline
\end{tabular}

a) “-”表示原文未提供相关数据 

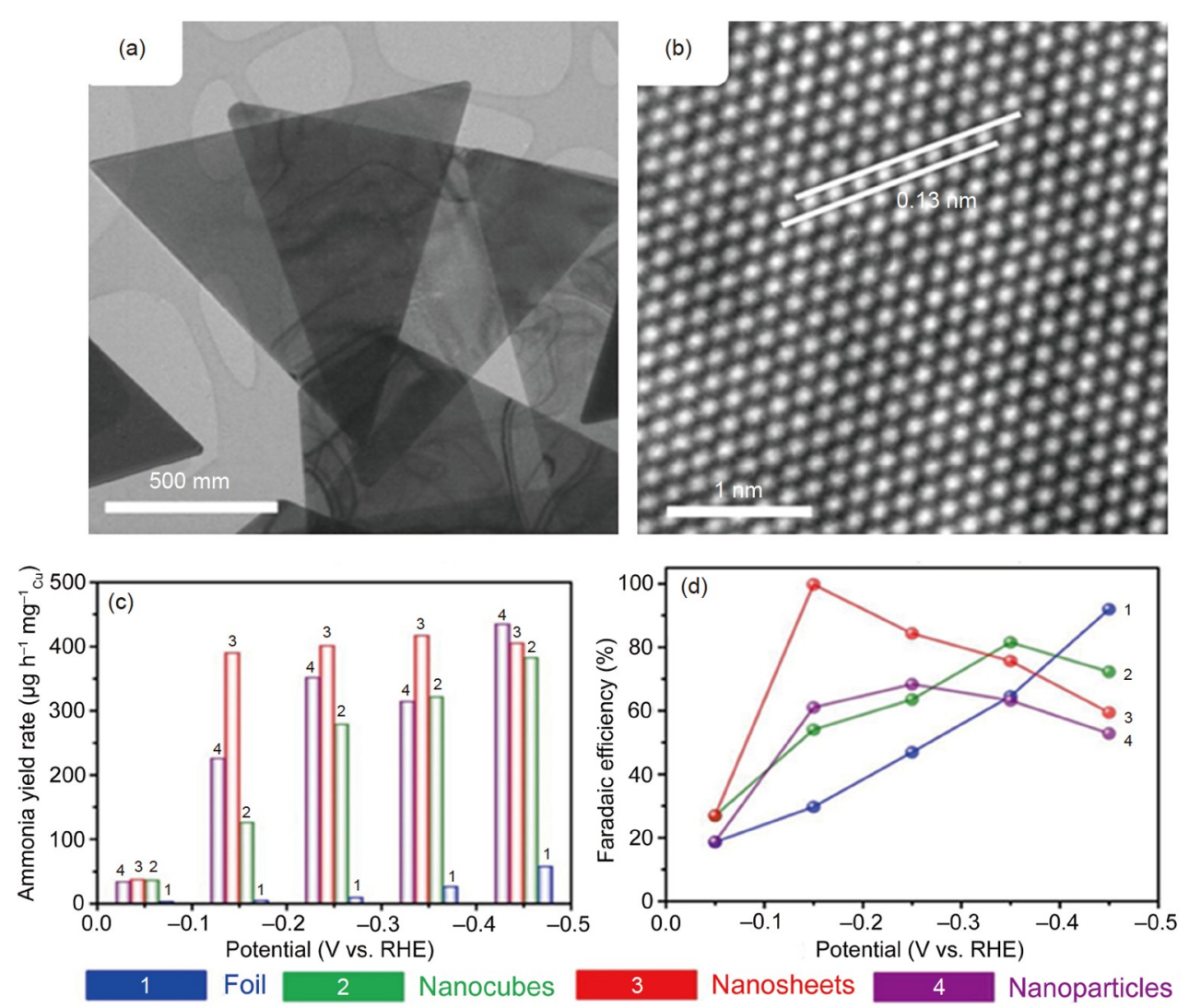

图 2 (网络版彩色)铜纳米片结构及电催化性能表征 ${ }^{[3]}$. (a) 铜纳米片TEM图; (b) 铜纳米片HRTEM图; 各类铜催化剂在不同电压下生成氨的产 率(c)和法拉第效率(d). Copyright $\odot 2020$ Tsinghua University Press and Springer-Verlag GmbH Germany

Figure 2 (Color online) The characterization of structure and catalytic performance for $\mathrm{Cu}$ nanosheets ${ }^{[3]}$. (a) TEM image of $\mathrm{Cu}$ nanosheets; (b) HRTEM image of $\mathrm{Cu}$ nanosheets basal plane; ammonia yield rates (c) and Faradaic efficiencies (d) of various $\mathrm{Cu}$ catalysts for ammonia production at different applied potentials. Copyright $\odot 2020$ Tsinghua University Press and Springer-Verlag GmbH Germany

$\mathrm{Cu}$ 纳米带电极对硝酸盐的还原率在 $60 \mathrm{~min}$ 时达到 $100 \%$. 而在相同条件下, 泡沫铜电极的硝酸盐还原率仅 为 $2.6 \%$. 实验证实, $3 \mathrm{D}-\mathrm{Cu}$ 纳米带能显著降低包括析氢 反应和氧还原反应在内的竞争反应的速率. 因此, 改变 铜的形貌结构可以提高电催化硝酸盐还原的速率及产 物的选择性. 但是, 单质铜的稳定性和耐腐蚀性差, 不 易长期用来进行电催化硝酸盐还原.

\section{2 单原子铜电催化硝酸盐还原}

近年来，单原子铜催化剂作为一种新型高效电催 化剂引起研究者的关注. 它是一类将孤立分散的金属 铜原子负载到载体表面，原子利用率接近 $100 \%$ ，在催 化反应中表现出高的活性、选择性和稳定性的新型催 化剂. 研究方向主要集中在：(1) 加强单原子与载体的 结合; (2) 实现单原子催化剂的高负载量, 从而进一步 提高硝酸盐的还原率.
$\mathrm{Zhu}$ 等人 ${ }^{[48]}$ 首次报道了将单原子铜负载到氮化碳 纳米片 $(\mathrm{Cu}-\mathrm{N}-\mathrm{C})$ 上作为催化剂进行电催化硝酸盐还原 为氨的研究. 在 $800^{\circ} \mathrm{C}$ 下热解, 分散的铜原子与碳纳米 片中的 $\mathrm{N}$ 进行配位, 合成了一种由碳纳米片与单原子铜 组成的金属-氮-碳电催化剂(Cu-N-C-800)(图3(a)). 动力 学和催化性能实验结果表明, 反应 $12 \mathrm{~h}$ 后, Cu-N-C-800 电催化 $\mathrm{NO}_{3}{ }^{-}$的还原率最高, $\mathrm{NO}_{2}^{-}$的还原率也最高, 说明 $\mathrm{Cu}-\mathrm{N}-\mathrm{C}-800$ 比 $\mathrm{Cu}$ 纳米颗粒和 $\mathrm{Cu}$ plate-800电催化硝酸 盐还原的活性高，并明显抑制了亚硝酸盐中间体向溶 液的释放(图3(b), (c)), 其 $\mathrm{NO}_{3}{ }^{-}$还原率为 $97.3 \%, \mathrm{NH}_{3}$ 的 选择性为 $80.5 \%$. 经过 20 个循环实验后, 依然保持较高 的 $\mathrm{NO}_{3}{ }^{-}$还原率，证明其具有良好的稳定性. 同时, 将实 验结果与密度泛函理论(DFT)计算相结合发现(图3(d)), $\mathrm{NO}_{3}{ }^{-}$对 $\mathrm{Cu}(111)$ 的计算吸附能为 $6.13 \mathrm{eV}$, 远高于 $\mathrm{Cu}-\mathrm{N}_{2}$ $(-4.06 \mathrm{eV})$ 和 $\mathrm{Cu}-\mathrm{N}_{4}(-2.50 \mathrm{eV})$ ，表明单个铜原子中的 $\mathrm{Cu}-\mathrm{N}_{x}$ 活性位点比 $\mathrm{Cu}$ 纳米颗粒中的 $\mathrm{Cu}(111)$ 更有利于 
(a)
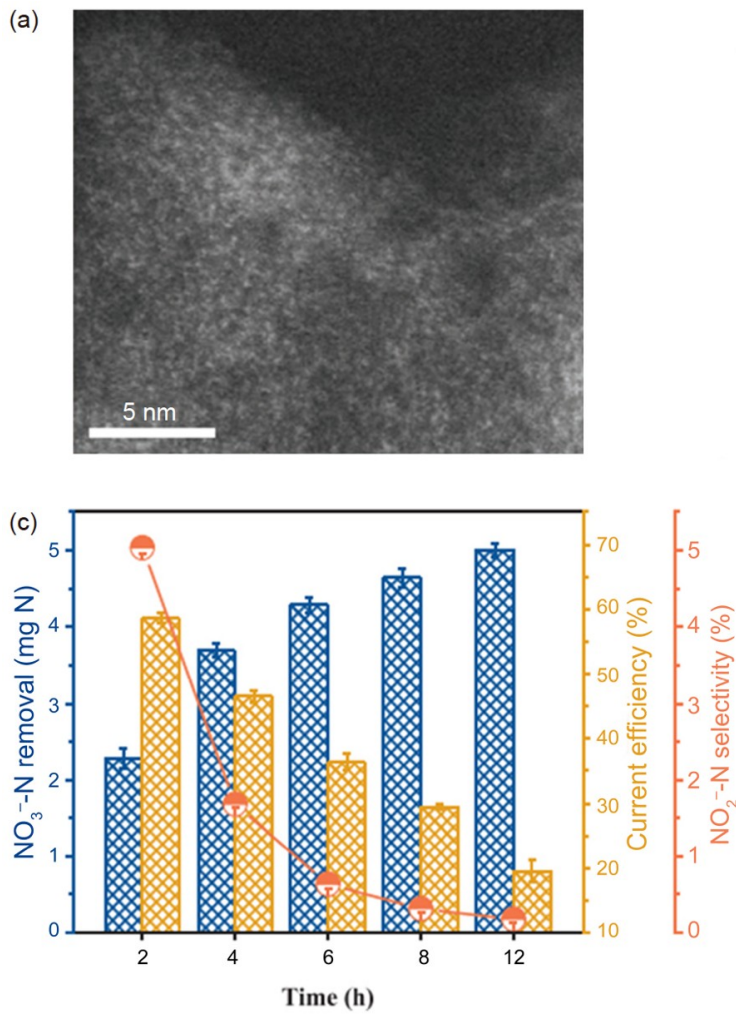
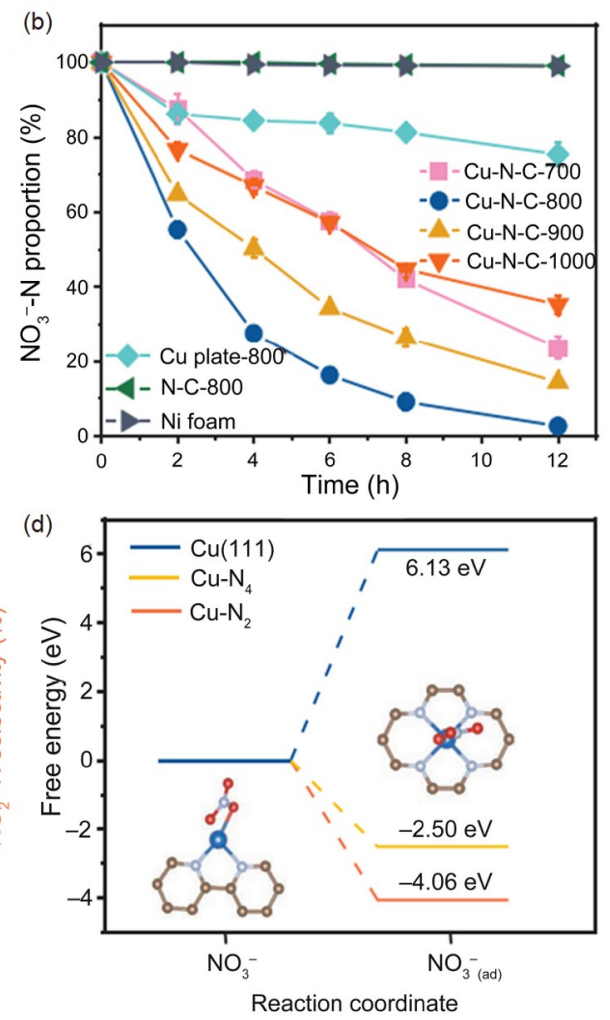

图 3 (网络版彩色)单原子铜催化剂结构以及催化性能表征 ${ }^{[48]}$. (a) Cu-N-C-800催化剂的TEM图; (b) 不同电解时间下, 硝酸盐还原 $\mathrm{NO}_{3}{ }^{-}$余量; (c) 不同电解时间下, $\mathrm{Cu}-\mathrm{N}-\mathrm{C}-800$ 电催化硝酸盐还原过程中的 $\mathrm{NO}_{3}^{-}$去除率、 $\mathrm{NO}_{2}^{-}$的选择性和电流效率; (d) DFT计算结果. Copyright $\mathrm{C} 2020$ Wiley$\mathrm{VCH} \mathrm{GmbH}$

Figure 3 (Color online) The characterization of structure and catalytic performance for $\mathrm{Cu}$ single-atom catalyst ${ }^{[48]}$. (a) TEM image of Cu-N-C-800 catalyst; (b) nitrate reduction of $\mathrm{NO}_{3}{ }^{-}$residual amount in different electrolysis time; (c) the $\mathrm{NO}_{3}^{-}$removal rate, $\mathrm{NO}_{2}{ }^{-}$selectivity and current efficiency of $\mathrm{Cu}-\mathrm{N}-\mathrm{C}-800$ electrocatalytic nitrate reduction process under different electrolytic time; (d) DFT calculation results. Copyright $(\mathrm{C} 2020 \mathrm{Wiley-VCH}$ $\mathrm{GmbH}$

$\mathrm{NO}_{3}{ }^{-}$的吸附, 而且 $\mathrm{NO}_{2}{ }^{-}$的吸附具有相同的趋势. 这种牢 固的结合有利于提高硝酸盐转化为 $\mathrm{NH}_{3}$ 的速率.

因此，单原子铜催化剂具有最大的原子利用效率 和良好的催化性能，其优势包括：(1) 充分的活性原子 和低配位金属中心促进了活性物质的吸附和转化; (2) 均匀的活性位点和几何结构增强了产物的选择性; (3) 由于单个原子与周围配位原子之间的强相互作用，使 其稳定性显著增加. 目前, 单原子铜催化剂在电催化硝 酸盐还原方面的研究较少, 还需要更多的理论支撑, 同 时也为电催化硝酸盐还原领域的发展提供了一个新 思路.

\section{3 铜合金电催化硝酸盐还原}

异质金属元素合金化是改善基体金属表面化学性 质的有效途径. 因为金属之间具有电子协同效应, 多元 金属电极性能稳定, 具有抗腐蚀特性, 所以催化活性普
遍高于单金属, 引起研究者的广泛关注.

\subsection{1 二元铜合金催化剂}

二元铜合金是铜与另一种金属元素融合而成的具 有金属性质的物质. 两种金属结合后会发生晶格参数 的改变和价电子在新势场中的重排等一系列变化, 可 以显著提高电催化性能，包括活性、选择性和稳定性. 表2总结了二元铜合金电极在硝酸盐还原中的性能.

Wang 等人 ${ }^{[49]}$ 基于 $\mathrm{Cu}$ 电子结构优化得到的 $\mathrm{CuFe}$ 纳 米枝晶状(图4(a))催化剂用于硝酸盐还原产 $\mathrm{NH}_{3}$. 通过 DFT计算结果得知, $\mathrm{Fe}$ 的引人导致 $\mathrm{Cu}$ 的 $3 \mathrm{~d}$ 能带向深能 级迁移(图4(b)). 这种 $\mathrm{Cu} 3 \mathrm{~d}$ 电子的重新分布有利于调 节反应中间体的吸附能. 电催化硝酸盐还原动力学的 测试结果表明, 在 $-0.7 \mathrm{~V}\left(\mathrm{vs}\right.$. RHE)下, $\mathrm{Cu}_{49} \mathrm{Fe}_{1}$ 的动力学 电流密度为 $55.6 \mathrm{~mA} / \mathrm{cm}^{2}$ (图4(c)), 是 $\mathrm{Cu}$ 的 2.1 倍, 证实了 $\mathrm{Cu}_{49} \mathrm{Fe}_{1}$ 具有更优的催化活性. $\mathrm{H}$ 型电解池中进行催化 剂的选择性测试结果表明, $\mathrm{Cu}_{49} \mathrm{Fe}_{1}$ 催化剂在 $-0.74 \mathrm{~V}$ 的 
表 2 二元铜合金电极在硝酸盐还原中的性能总结 ${ }^{\text {a) }}$

Table 2 Summary of the performance of binary copper alloy electrodes in nitrate reduction

\begin{tabular}{|c|c|c|c|c|c|c|}
\hline 阴极材料 & 阴极面积 $\left(\mathrm{cm}^{2}\right)$ & 电解液 & $\mathrm{NO}_{3}$ 转化率 $(\%)$ & 选择性(\%) & 时间(h) & 文献 \\
\hline $\mathrm{Cu}_{49} \mathrm{Fe}_{1}$ & 1 & $200 \mathrm{mg} / \mathrm{L} \mathrm{NO}_{3}^{-}(40 \mathrm{~mL})$ & 96.9 & $86.8\left(\mathrm{NH}_{4}^{+}\right)$ & 2 & [49] \\
\hline $\mathrm{Cu} / \mathrm{Ti}$ & 25 & $50 \mathrm{mg} / \mathrm{L} \mathrm{NO}_{3}^{-}(100 \mathrm{~mL})$ & 70 & - & 1.5 & [50] \\
\hline $\mathrm{Cu}_{50} \mathrm{Ni}_{50}$ & - & $100 \mathrm{mmol} / \mathrm{L} \mathrm{KNO}_{3}+1 \mathrm{~mol} / \mathrm{L} \mathrm{KOH}$ & - & $15\left(\mathrm{NH}_{4}^{+}\right)$ & 12 & [51] \\
\hline $\mathrm{Cu} / \mathrm{Zn} / \mathrm{TiO}_{2}$ & 25 & $50 \mathrm{mg} / \mathrm{L} \mathrm{NO}_{3}^{-}+0.5 \mathrm{~g} / \mathrm{L} \mathrm{Na}_{2} \mathrm{SO}_{4}(100 \mathrm{~mL})$ & 97.5 & - & 1.5 & {$[52]$} \\
\hline $\mathrm{Cu} / \mathrm{Zn}$ & 75 & $100 \mathrm{mg} / \mathrm{L} \mathrm{NO}_{3}^{-}(400 \mathrm{~mL})$ & 90 & - & 3 & [53] \\
\hline $\mathrm{CuNi}$ & 12 & $100 \mathrm{mg} / \mathrm{L} \mathrm{NO}_{3}^{-}(100 \mathrm{~mL})$ & 99 & - & 1 & [54] \\
\hline $\mathrm{CuSn}_{6}$ & - & $1 \mathrm{mmol} / \mathrm{L} \mathrm{NO}_{3}^{-}$ & 59 & $80.37\left(\mathrm{~N}_{2}\right)$ & 20 & {$[55]$} \\
\hline $\mathrm{Cu}_{80} \mathrm{Ni}_{20}$ & 6 & $20 \mathrm{mmol} / \mathrm{L} \mathrm{NaNO}_{3}+1 \mathrm{~mol} / \mathrm{L} \mathrm{NaOH}$ & 50 & $88\left(\mathrm{NH}_{4}^{+}\right)$ & 8 & {$[56]$} \\
\hline $\mathrm{Cu}_{70} \mathrm{Ni}_{30}$ porous & 3 & $0.1 \mathrm{~mol} / \mathrm{L} \mathrm{NaNO}_{3}+1 \mathrm{~mol} / \mathrm{L} \mathrm{NaOH}(100 \mathrm{~mL})$ & 86.8 & $95.9\left(\mathrm{NH}_{4}^{+}\right)$ & 8 & {$[57]$} \\
\hline $\mathrm{Cu}_{70} \mathrm{Zn}_{30}$ porous & 3 & $0.1 \mathrm{~mol} / \mathrm{L} \mathrm{NaNO}_{3}+1 \mathrm{~mol} / \mathrm{L} \mathrm{NaOH}(100 \mathrm{~mL})$ & 100 & - & 8 & [58] \\
\hline $\mathrm{Pd}_{80}-\mathrm{Cu}_{20}$ & - & $0.1 \mathrm{~mol} / \mathrm{L} \mathrm{NaNO}_{3}+1 \mathrm{~mol} / \mathrm{L} \mathrm{NaOH}$ & 79 & $87\left(\mathrm{NH}_{4}^{+}\right)$ & 6 & [59] \\
\hline $\mathrm{Ti} / \mathrm{Cu}_{5} \mathrm{ZnO}_{x}$ & 4 & $150 \mathrm{mg} / \mathrm{L} \mathrm{NaNO}{ }_{3}+500 \mathrm{mg} / \mathrm{L} \mathrm{NaCl}$ & 92.3 & $33.7\left(\mathrm{~N}_{2}\right)$ & 6 & {$[60]$} \\
\hline $\mathrm{Pd}-\mathrm{Cu} / \gamma \mathrm{Al}_{2} \mathrm{O}_{3}$ & - & $50 \mathrm{mg} / \mathrm{L} \mathrm{NO}_{3}^{-}(100 \mathrm{~mL})$ & 98.7 & $80.37\left(\mathrm{~N}_{2}\right)$ & 1 & [61] \\
\hline
\end{tabular}

a) “-”表示原文未提供相关数据
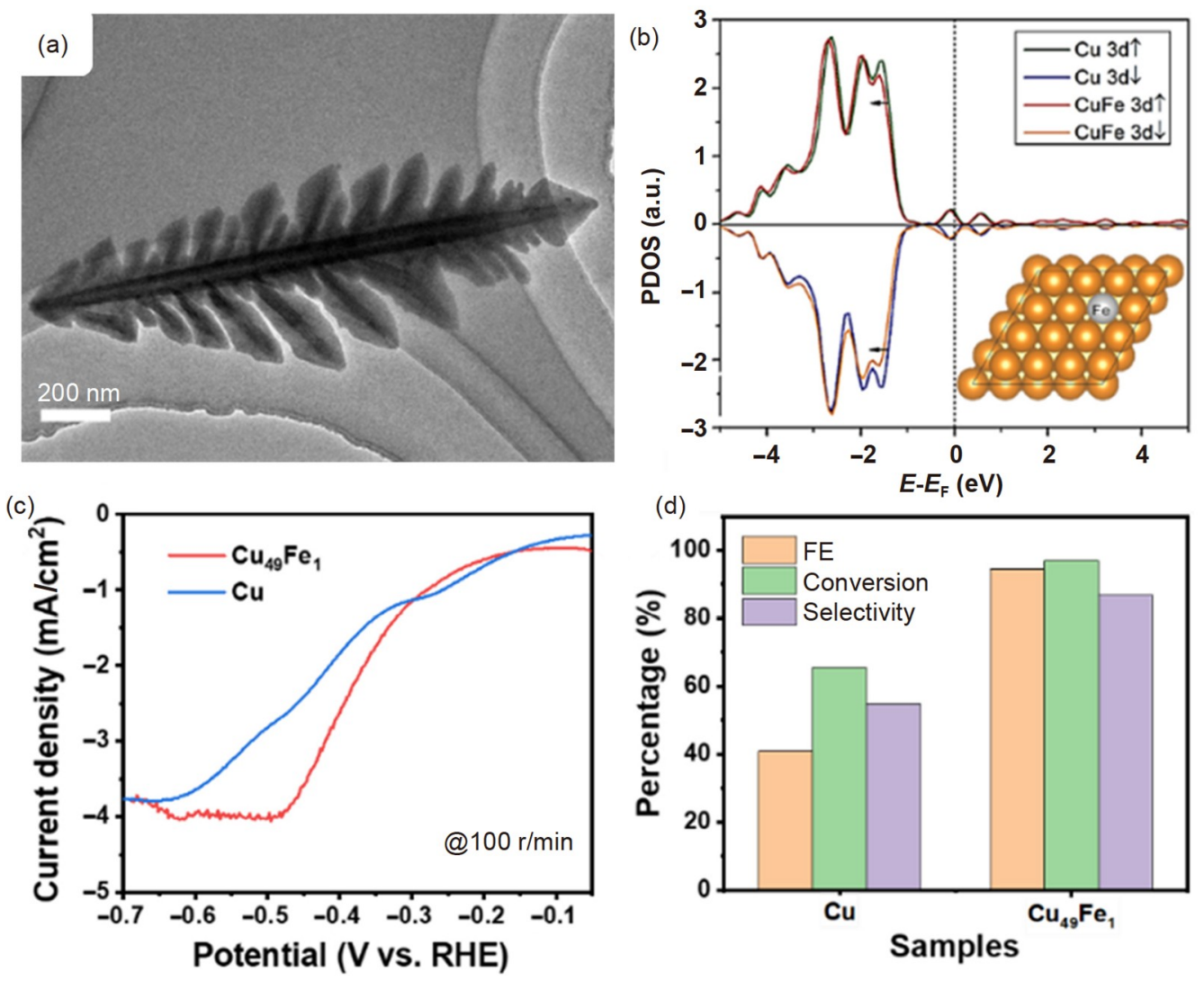

图 4 (网络版彩色)铜铁合金结构及催化性能表征 ${ }^{[49]}$. (a) $\mathrm{Cu}_{49} \mathrm{Fe}_{1}$ 纳米枝晶TEM图; (b) $\mathrm{Cu}_{49} \mathrm{Fe}_{1}$ 的DFT计算结果; (c) $\mathrm{Cu}$ 和 $\mathrm{Cu}_{49} \mathrm{Fe}_{1}$ 在旋转环盘电极 上的硝酸根还原极化曲线; (d) $\mathrm{Cu}$ 和 $\mathrm{Cu}_{49} \mathrm{Fe}_{1}$ 的法拉第效率、 $\mathrm{NO}_{3}{ }^{-}$转换率和 $\mathrm{NH}_{3}$ 的选择性. Copyright (c) $2021 \mathrm{Wiley-} \mathrm{VCH} \mathrm{GmbH}$

Figure 4 (Color online) The characterization of structure and catalytic performance for CuFe alloy ${ }^{[49]}$. (a) TEM image of $\mathrm{Cu}_{49} \mathrm{Fe}_{1}$ nanocrystal; (b) DFT calculation results of $\mathrm{Cu}_{49} \mathrm{Fe}_{1}$; (c) the polarization curves of nitrate reduction on the $\mathrm{Cu}$ and $\mathrm{Cu}_{49} \mathrm{Fe}_{1}$-modified RDEs; (d) Faradic efficiency, conversion rate of $\mathrm{NO}_{3}{ }^{-}$and $\mathrm{NH}_{3}$ selectivity over the $\mathrm{Cu}$ and $\mathrm{Cu}_{49} \mathrm{Fe}_{1}$. Copyright (C) 2021 Wiley-VCH GmbH 
电解电压时具有 $94.5 \%$ 的 $\mathrm{NH}_{3}$ 选择性，明显高于 $\mathrm{Cu}$ 的 $40.9 \%$, 证实了 $\mathrm{Cu}_{49} \mathrm{Fe}_{1}$ 良好的 $\mathrm{NH}_{3}$ 选择性(图4(d)). 与 $\mathrm{Li}$ 等人 ${ }^{[62]}$ 报道的 $\mathrm{Fe}$ 电极在电解 $4 \mathrm{~h}$ 后, 硝酸盐还原率为 $91 \%, \mathrm{NH}_{3}$ 选择性仅为 $28 \%$ 相比, $\mathrm{CuFe}$ 合金化后的电催 化硝酸盐还原性能远高于单质 $\mathrm{Cu}$ 和 $\mathrm{Fe}$, 而且 $\mathrm{NH}_{3}$ 选择 性有明显提升. $\mathrm{Liu}^{[50]}$ 制备了一种新型多孔结构的铜钛 合金双层纳米电极 $(\mathrm{Cu} / \mathrm{Ti})$, 其中 $\mathrm{Ti}$ 和 $\mathrm{Cu}$ 含量分别为 $80.72 \mathrm{wt} \%$ 和 $19.28 \mathrm{wt} \%$ (重量百分比), 具有厚而光滑的 孔壁. 电极表面的 $\mathrm{Ti}$ 纳米结构具有大的比表面积, 而 $\mathrm{Cu}$ 对硝酸盐还原具有很高的电催化活性，两者结合极大 提高了电催化硝酸盐还原的效率. 在 $0.5 \mathrm{mg} / \mathrm{L} \mathrm{Na}_{2} \mathrm{SO}_{4}$ 、 电流密度为 $30 \mathrm{~mA} / \mathrm{cm}^{2}$ 条件下电解 $90 \mathrm{~min}$ 后, 硝酸盐浓 度由最初的 $50.0 \mathrm{mg} / \mathrm{L}$ 降至 $14.1 \mathrm{mg} / \mathrm{L}$ (硝酸盐还原率为 $71.8 \%$ ). 而且在相同的初始硝酸盐浓度下, 随着电流密 度的增大, 硝酸盐还原率迅速升高. 与 $\mathrm{Li}$ 等人 ${ }^{[63]}$ 报道的 Ti电极在电解 $3 \mathrm{~h}$ 时, 硝酸盐的还原率仅为 $14.6 \%$ 相比, 电催化硝酸盐还原能力有了极大的提升. Wang等人 ${ }^{[51]}$ 通过调节原子中间的吸附态，增强铜镍合金 $\left(\mathrm{Cu}_{x} \mathrm{Ni}_{y}\right)$ 催 化剂催化硝酸盐还原生成 $\mathrm{NH}_{3}$ 的性能. 用 $\mathrm{Ni}$ 代替 $50 \%$ 的 $\mathrm{Cu}$ 生成 $\mathrm{Cu}_{50} \mathrm{Ni}_{50}$, 使其性能显著提升, 包括半波电位上 移了 $0.12 \mathrm{~V}$, 实现最佳 $\mathrm{NH}_{3}$ 法拉第效率的过电位降低了 $0.2 \mathrm{~V}$. 在碱性条件下 (pH 14), 与 $0 \mathrm{~V}$ (vs. RHE)的纯Cu相 比, $\mathrm{Cu}_{50} \mathrm{Ni}_{50}$ 合金催化剂的还原活性提高了6倍. 电子结 构研究表明, d带中心向费米能级上移, 该特征增强了 中间吸附能. 同时利用DFT计算也验证了这种关系, 由 于 $\mathrm{NO}_{3}{ }^{-}$在 $\mathrm{Cu}_{50} \mathrm{Ni}_{50}$ 表面的吸附能增强, 降低了过电位, 引人 $\mathrm{Ni}$ 原子会使电位相关阶跃 $(\mathrm{PDS})$ 从 $\mathrm{NO}_{3}{ }^{-}$吸附转变 为 $* \mathrm{NH}_{2}$ 加氢, 从而确定了 $\mathrm{NO}_{3}{ }^{-}$还原生成 $\mathrm{NH}_{3}$ 的路径. $\mathrm{Liu}$ 等人 ${ }^{[52]}$ 制备了一种新型铜锌氧化钛纳米管阵列 $(\mathrm{Cu}-$ $\mathrm{Zn}-\mathrm{TiO}_{2}-\mathrm{TNTA}$ )催化剂, 其结构为蜂窝状、海绵体状. $\mathrm{Cu}-\mathrm{Zn}$-TNTA纳米电极表面的Ti纳米结构使纳米电极 具有较大的比表面积, 与 $\mathrm{Cu}$ 和 Zn结合增强了硝酸盐电 催化还原产生 $\mathrm{N}_{2}$ 的活性. 在 $0.5 \mathrm{mg} / \mathrm{L} \mathrm{Na} \mathrm{SO}_{4}$ 、电流密 度为 $30 \mathrm{~mA} / \mathrm{cm}^{2}$ 的条件下, 用 $\mathrm{Cu}-\mathrm{Zn}-\mathrm{TiO}_{2}-\mathrm{TNTA}$ 纳米电 极电催化硝酸盐的还原率为 $97.5 \%$. 而且在体系中再加 人 $0.3 \mathrm{~g} / \mathrm{L} \mathrm{NaCl}$ 后, 硝酸盐完全还原, 处理后的溶液中未 检测到 $\mathrm{NH}_{3}$. 在相同的电流密度下, 随着初始硝酸盐浓 度的增加, 硝酸盐还原率略有下降; 在相同的初始硝酸 盐浓度下, 随着电流密度的增加, 硝酸盐还原率提高. 同时, $\mathrm{pH}$ 的改变对 $\mathrm{Cu}-\mathrm{Zn}-\mathrm{TiO}_{2}-\mathrm{TNTA}$ 纳米电极催化硝 酸盐还原的影响不大, 而温度的改变对硝酸盐的还原 有较强的影响.

\subsection{2 三元铜合金催化剂}

近几年，研究者对三元铜合金电催化剂的关注度 逐渐提高, 他们发现多种金属结合能够更好地优化催 化剂的电子结构, 促进硝酸根的吸附和还原.

Zhang等人 ${ }^{[64]}$ 报道了 $\mathrm{Cu} 、 \mathrm{Ni} 、 \mathrm{Zn}$ 三种金属组成铜 镍锌 $\left(\mathrm{Cu}_{x} \mathrm{Ni}_{y} \mathrm{Zn}_{z}\right)$ 合金催化剂进行硝酸盐还原. 正交实验 确定了 $\mathrm{Cu}_{x} \mathrm{Ni}_{y} \mathrm{Zn}_{z}$ 合金电极电催化硝酸盐还原的最佳条 件: $8 \mathrm{~mA} / \mathrm{cm}^{2}$ 电流密度、 $2.0 \mathrm{~g} / \mathrm{L} \mathrm{NaCl}$, 当初始 $\mathrm{NO}_{3}{ }^{-}$浓 度为 $100 \mathrm{mg} / \mathrm{L}$ 时, 初始 $\mathrm{pH}$ 为 3.0. $\mathrm{Cu}_{60} \mathrm{Ni}_{15} \mathrm{Zn}_{25}$ 在最佳条 件下的硝酸盐还原率为 $63.5 \%$. 与报道的 $\mathrm{Li}$ 单质、 $\mathrm{Ni}$ 电 极 ${ }^{[62]}$ 相比 $(4 \mathrm{~h}$ 后的硝酸盐还原率仅为 $36 \%), \mathrm{Cu}_{x} \mathrm{Ni}_{y} \mathrm{Zn}_{z}$ 合金电极的硝酸盐还原率提高了 1.76 倍. Gao等人 ${ }^{[65]}$ 报 道了利用电沉积法将 $\mathrm{Cu}-\mathrm{Sn}-\mathrm{Bi}$ 镀在 $\mathrm{Ti}$ 基上, $\mathrm{Cu}-\mathrm{Sn}-\mathrm{Bi}$ 电 极的涂层颗粒比其他电极更加紧凑，可以提供更多的 反应点, 增加了电极的电化学活性. 研究发现, -0.6 $0.8 \mathrm{~V}\left(\mathrm{vs} . \mathrm{Hg} / \mathrm{HgO}\right.$ ) 时, $\mathrm{NO}_{3}{ }^{-}$将吸附于 $\mathrm{Cu}-\mathrm{Sn}-\mathrm{Bi}$ 电极上的 $\mathrm{NO}_{3}{ }^{-}$转化为 $\mathrm{NO}_{2}{ }^{-}$, 当电压达到 $-1.5 \mathrm{~V}(\mathrm{vs} . \mathrm{Hg} / \mathrm{HgO}$ ) 时, $\mathrm{NO}_{2}$ 转化为 $\mathrm{NH}_{3}$. 结果表明, $100 \mathrm{mg} / \mathrm{L} \mathrm{NO}_{3}^{-}$、电流密 度为 $6 \mathrm{~mA} / \mathrm{cm}^{2}$ 的条件下, 硝酸盐还原率高达 $88.43 \%, \mathrm{~N}_{2}$ 的选择性可以达到 $77.80 \%$. 当Bi的含量为 $3.18 \%$ 时，没 有发现明显的电流衰减, 显示出良好的稳定性. Bi的引 人不仅增强了硝酸盐还原的电催化活性，而且提高了 催化剂的稳定性, 实现了高效电催化硝酸盐还原.

因此，铜合金催化剂不仅可以提高硝酸盐的催化 效率和稳定性，而且通过探索两种或多种金属之间的 电子结构排布与电催化活性的关系, 提高了电催化硝 酸盐还原率，同时也为高性能合金电催化剂的设计开 辟了一条新途径.

\section{4 铜基复合材料电催化硝酸盐还原}

\subsection{1 铜与非金属复合催化剂}

由于非金属材料具有成本低、机械强度高、电子 结构可调性强、耐久性好等优点, 也被用作电化学还 原硝酸盐的阴极. 其与铜、铜合金相结合提高了电极 中活性结合位点、电子的转移速率, 促进了电催化硝 酸盐的还原.

Couto等人 ${ }^{[66]}$ 报道了利用等离子处理负载 $\mathrm{Cu}$ 纳米 颗粒的嗍金刚石薄膜 $(\mathrm{BDD} / \mathrm{Cu})$ 用于电催化硝酸盐还 原. $\mathrm{BDD} / \mathrm{Cu}$ 分别采用氢、氧等离子体进行处理, 其中, 氧等离子体处理的样品与氢等离子处理的样品相比, 其硝酸盐还原的性能和重现性更好. 研究者认为这种 差异主要源自 $\mathrm{Cu}$ 纳米粒子表面预处理所产生的官能团 
不同. 其中, 氧等离子体处理对 $\mathrm{Cu}$ 纳米粒子在BDD表面 的固定起着重要作用, 氧官能团可以作为 $\mathrm{Cu}$ 团簇的针 定点, 增强BDD和 $\mathrm{Cu}$ 之间的界面附着力, 极大提高了 电催化硝酸盐还原能力和电极的稳定性. 而氢官能团 等离子体处理后, 使得 $\mathrm{Cu}$ 镀层均匀, 颗粒密度高, 仅仅 提高了电极的电导率, 对于电催化硝酸盐还原能力的 提升效果并不明显. Chen等人 ${ }^{[67]}$ 将钯铜合金负载在类 似珊瑚结构的 $\mathrm{N}$ 掺杂多孔碳 $\left(\mathrm{Pd}_{x} \mathrm{Cu}_{y} @ \mathrm{~N}-\mathrm{pC}\right)$ 复合材料 上, 用于电催化硝酸盐还原. N-pC的多孔结构带来较大 的比表面积, 与钯铜合金 $\left(\mathrm{Pd}_{x} \mathrm{Cu}_{y}\right)$ 相互作用, 优化 $\mathrm{Cu}$ 的 $\mathrm{d}$ 带电子结构, 增加了导电性, 使得复合催化剂 $\mathrm{Pd}_{4} \mathrm{Cu}_{4} @$ $\mathrm{N}-\mathrm{pC}$ 具有优良的电催化硝酸盐还原能力，硝酸盐还原 率高达 $95 \%$ ，氮选择性高达 $80 \%$. Yin等人 ${ }^{[17]}$ 报道了一 种铜纳米颗粒 $(\mathrm{Cu} N \mathrm{NPS})$ 与还原氧化石墨烯( $\mathrm{rGO})$ 相结 合的复合电极材料用于电催化硝酸盐还原. rGO具有 高比表面积 $\left(2965 \mathrm{~m}^{2} / \mathrm{g}\right)$ 、高导电率和良好的稳定性. 当其均匀负载 $\mathrm{Cu} N \mathrm{NPS}$ 形成 $\mathrm{Cu} / \mathrm{rGO} / \mathrm{GP}$ 电极, 其具有较 高的电子转移速率、较高的吸附容量和较大的电化学 活性比表面积, 能有效地增强电催化硝酸盐还原能力,
使硝酸盐还原率高达 $96.8 \%$ ，且 $\mathrm{NH}_{3}$ 的选择性为 $29.9 \%$ (图5). Zhang等人 ${ }^{[68]}$ 将铜钯纳米颗粒负载于碳纳米管 (CNTs/Cu5-Pd5), 制备成复合催化剂用于电催化硝酸 盐还原. 电化学分析结果表明, CNTs/Cu5-Pd5相对于 $\mathrm{Cu} 5-\mathrm{Pd} 5$ 催化剂具有更好的 $\mathrm{NO}_{3}{ }^{-}$转化率和 $\mathrm{N}_{2}$ 选择性, $\mathrm{CNTs} / \mathrm{Cu} 5-\mathrm{Pd} 5$ 电催化硝酸盐转化率为 $32.52 \%, \mathrm{~N}_{2}$ 选择 性为 $42.55 \%$. 虽然碳纳米管对硝酸盐还原反应几乎没 有催化活性, 也不会改变双金属的沉积量和组成, 但是 碳纳米管促进了双金属粒子的高分散性，从而显著提 升电极的电催化活性和稳定性. 这也证明了铜和钯在 催化硝酸盐还原方面具有良好的协同作用.

因此，非金属材料与具有硝酸盐还原催化活性的 铜基材料结合形成复合催化剂，一方面可以利用非金 属材料高的比表面积均匀分散活性催化剂，防止团聚; 另一方面，它们之间的相互作用能够有效提升催化 活性.

\section{4 .2 铜及氧化物复合催化剂}

铜氧化物材料因其析氢的有效抑制作用已被应用 于电催化二氧化碳还原中，而且电还原过程可能会诱
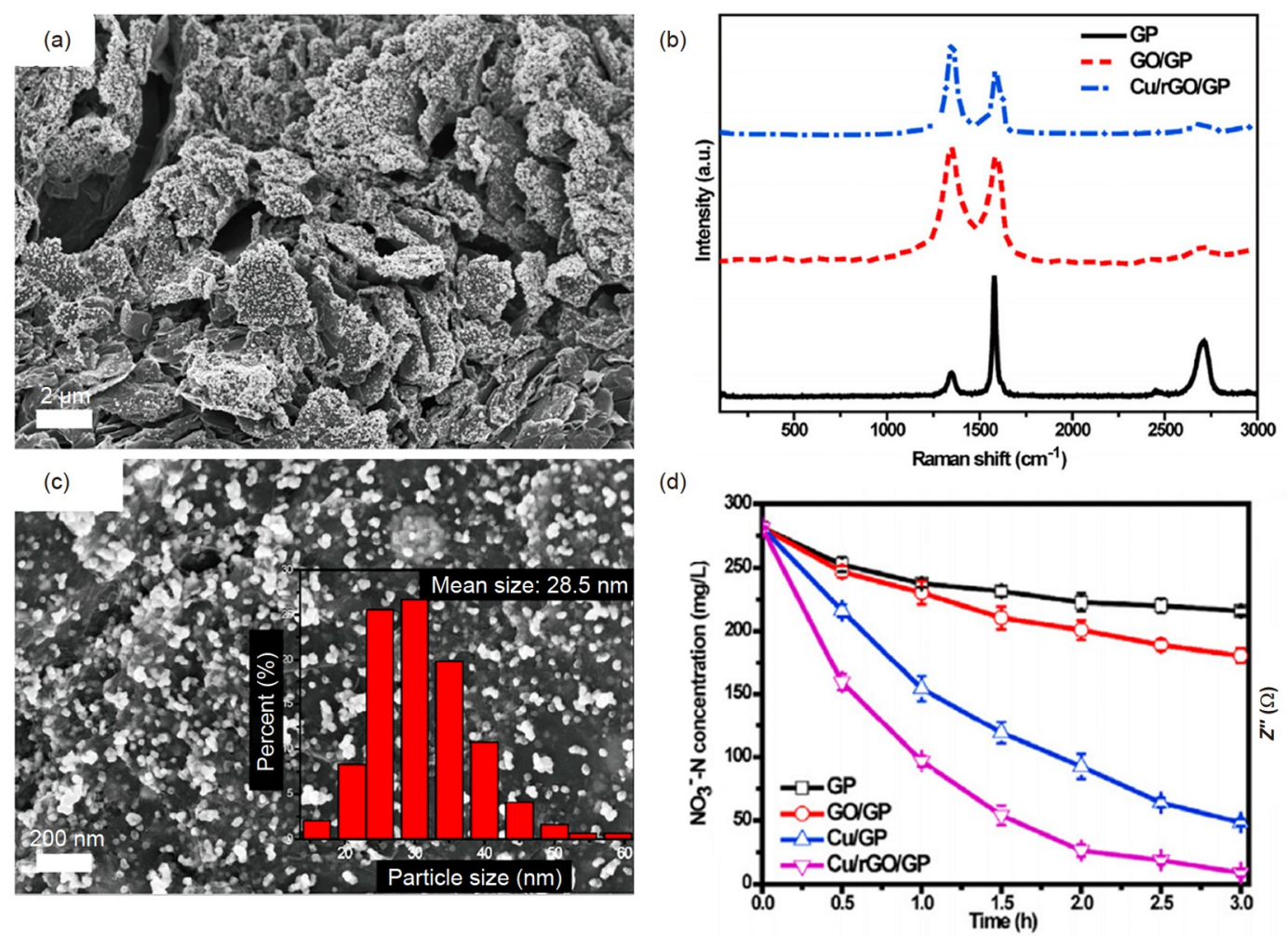

图 5 (网络版彩色) $\mathrm{Cu} / \mathrm{rGO} / \mathrm{GP}$ 结构及催化性能表征 ${ }^{[17]}$. (a), (c) $\mathrm{Cu} / \mathrm{rGO} / \mathrm{GP}$ 的SEM图; (b) 拉曼光谱图; (d) 硝酸盐浓度随时间变化图. Copyright (C) 2019 Elsevier

Figure 5 (Color online) The characterization of structure and catalytic performance for $\mathrm{Cu} / \mathrm{rGO} / \mathrm{GP}{ }^{[17]}$. (a), (c) The SEM images of Cu/rGO/GP; (b) Raman spectra; (d) time-dependent concentration change of $\mathrm{NO}_{3}^{-}$. Copyright $(C) 2019$ Elsevier 
导催化材料的结构演变. 因此, 揭示催化过程中活性相 的来源, 对电催化硝酸盐还原具有重要意义.

Wang等人 ${ }^{[21]}$ 报道了 $\mathrm{CuO}$ 纳米线阵列 $(\mathrm{CuO}$ NWAs $)$ 是电催化硝酸盐还原为 $\mathrm{NH}_{3}$ 的有效阴极材料, 经过电 化学还原后形成 $\mathrm{Cu} / \mathrm{Cu}_{2} \mathrm{O}$ 纳米阵列 $\left(\mathrm{Cu} / \mathrm{Cu}_{2} \mathrm{O}\right.$ NWAs $)$, 其催化性能如图6所示. $\mathrm{Cu} / \mathrm{Cu}_{2} \mathrm{O} \quad \mathrm{NWAs}$ 在 $\mathrm{NO}_{3}{ }^{-}$存在下 的电流密度比不含 $\mathrm{NO}_{3}{ }^{-}$的体系明显增加(图6(a)), 电压 为 $-0.55 \sim 0.95 \mathrm{~V}, \mathrm{NO}_{3}{ }^{-}$的转化率逐渐增加, 而法拉第效 率在 $-0.85 \mathrm{~V}$ 时达到最大值 $95.8 \%$ (图6(b)). 其具有良好 的稳定性，氨的产率和法拉第效率在 6 次循环后没有明 显的衰减(图6(c)). 考虑到纯 $\mathrm{Cu}_{2} \mathrm{O}$ 在电还原过程中不稳
定并且部分转化为 $\mathrm{Cu}$, 于是将其与铜网和 $\mathrm{Cu}$ NWAs对 电催化硝酸盐还原的性能进行了比较，表明其具有良 好的法拉第效率(95.8\%)和氨选择性(81.2\%)(图6(d)).

因此, $\mathrm{CuO} N W A s$ 电化学转化为 $\mathrm{Cu} / \mathrm{Cu}_{2} \mathrm{O} N W A s$, 其 作为硝酸盐还原的活性相, 首先吸附形成 $* \mathrm{NO}_{3}$, 总能量 降低, $* \mathrm{NO}_{3}$ 的 $\mathrm{O}$ 键一步自发裂解生成 $* \mathrm{NO}_{2}$ 和 $* \mathrm{NO}$, 然后 *NO发生氢化反应, 形成* $\mathrm{NOH}$. 随后, $* \mathrm{NOH}$ 氢化生成 $* \mathrm{NH}_{2} \mathrm{OH}$, 再转化为 $* \mathrm{NH}_{3}$. 最后 $* \mathrm{NH}_{3}$ 从催化剂中脱出. 与 $\mathrm{Cu}$ 相比, $\mathrm{Cu} / \mathrm{Cu}_{2} \mathrm{O}$ 有利于生成 $\mathrm{NOH}$ 中间体, 其电子云 密度和电子转移速度发生改变(图6(e)). 因此, 在 $\mathrm{Cu} / \mathrm{Cu}_{2} \mathrm{O}$ NWAs上，反应更容易进行，为硝酸盐还原中构建高效
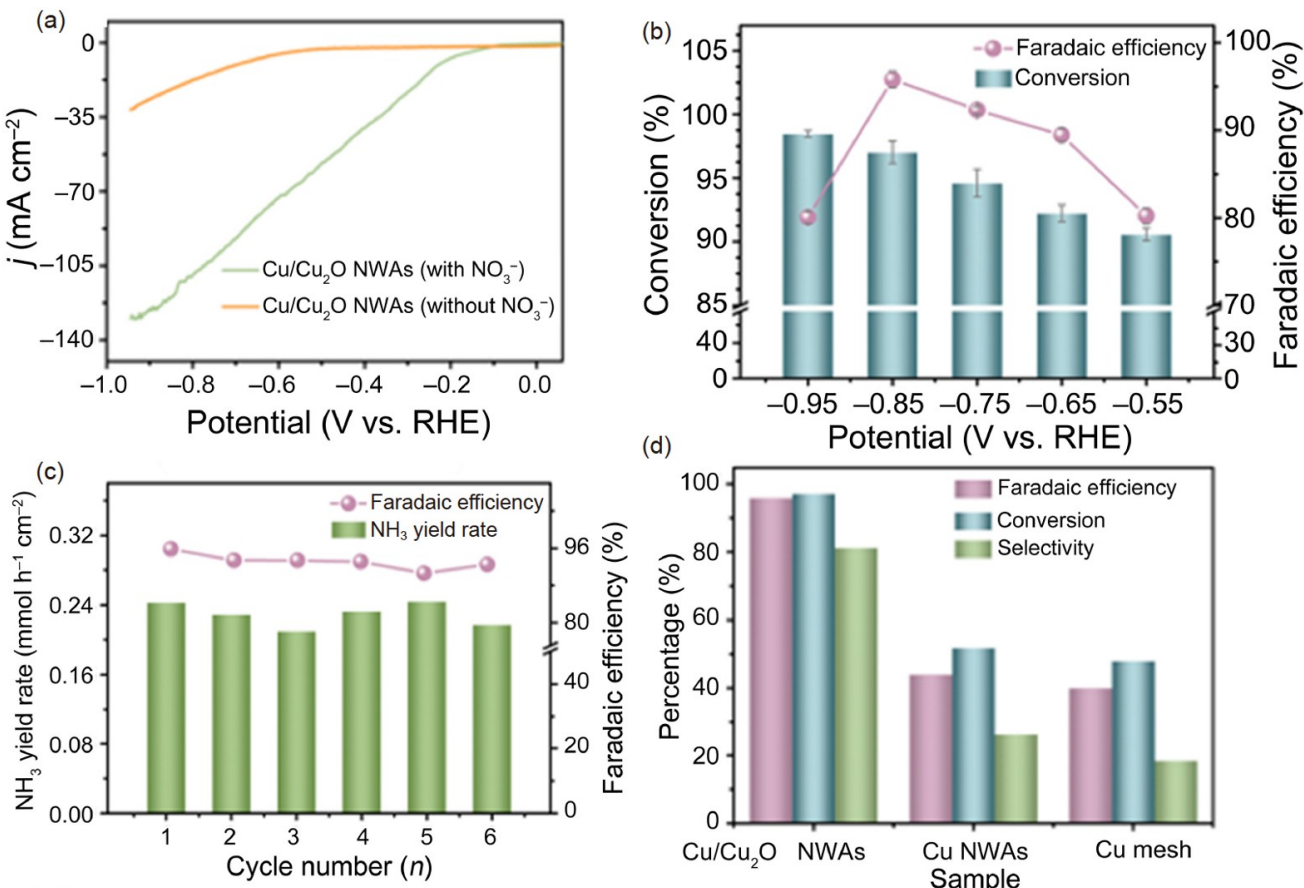

(d) Potential (V vs. RHE)
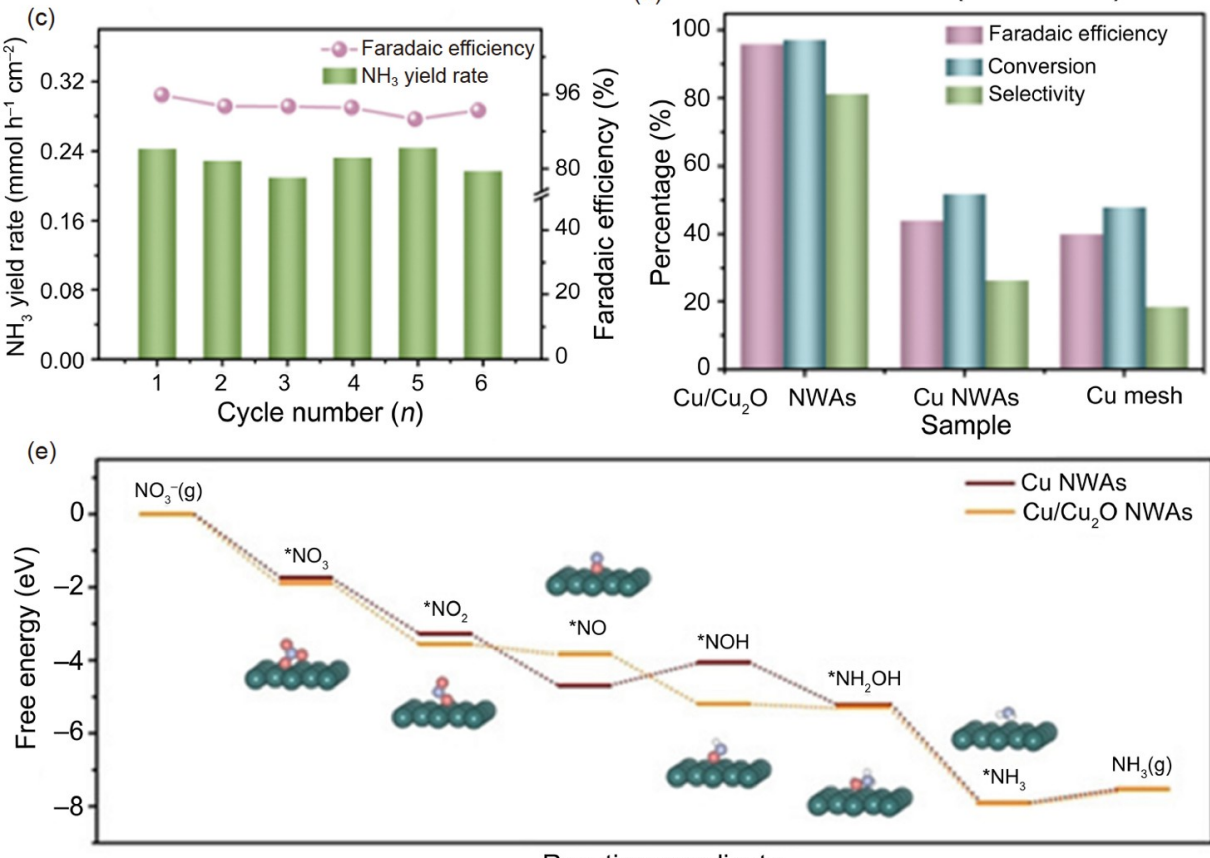

Reaction coordinate

图 6 (网络版彩色) $\mathrm{Cu} / \mathrm{Cu}_{2} \mathrm{O}$ NWAs的催化性能表征 ${ }^{[21]}$. (a) $\mathrm{Cu} / \mathrm{Cu}_{2} \mathrm{O}$ NWAs的极化曲线( $\mathrm{LSV}$ curves); (b) 不同电压下的硝酸盐转化率和法拉第效 率; (c) -0.85 V下的循环性能测试; (d) 法拉第效率、氨的选择性和硝酸盐转化率; (e) 自由能图. Copyright (C) 2020 Wiley-VCH Verlag GmbH \& Co. KGaA, Weinheim

Figure 6 (Color online) The characterization of catalytic performance for $\mathrm{Cu} / \mathrm{Cu}_{2} \mathrm{O}$ NWAs ${ }^{[21]}$. (a) LSV curves of $\mathrm{Cu} / \mathrm{Cu}{ }_{2} \mathrm{O} \mathrm{NWAs}$; (b) potentialdependent Faradaic efficiency of ammonia and conversion rate of nitrate; (c) the consecutive recycling test at $-0.85 \mathrm{~V}$; (d) Faradaic efficiency and selectivity of ammonia and conversion rate of nitrate; (e) free energy diagram. Copyright (C) 2020 Wiley-VCH Verlag GmbH \& Co. KGaA, Weinheim 
合成氨的电催化剂开辟了新途径.

\section{3 总结与展望}

本文主要综述了铜基材料催化剂电催化硝酸盐还 原的研究进展，分别从单质铜、单原子铜、铜合金和 铜基复合材料电催化硝酸盐还原的角度分析了结构与 其选择性和催化活性之间的关系. 首先, 通过改变单质 铜的形貌调控其表面缺陷和暴露的晶面, 进而优化电 催化硝酸盐的还原性. 由于单质铜不耐腐蚀以及催化 稳定性差，因此不易长期用来进行硝酸盐还原. 其次, 单原子铜电极材料在催化反应中表现出高的活性和选 择性. 但是如何实现单原子催化剂的高负载量, 是研究 者亟须突破的问题. 然后, 铜合金极大改进了催化剂稳 定性的问题，同时由于金属之间的协同电子效应，多元 金属电极性能稳定且具有抗腐蚀特性，催化活性高于 普通单金属. 但是铜合金, 尤其是掺杂贵重金属的铜合 金, 其成本相应提高, 不利于大面积的工业化推广. 最 后, 铜与非金属材料载体形成复合催化剂, 可结合非金 属材料成本低、机械强度高、电子结构可调性强、耐 久性好等优点, 均匀分散铜纳米催化剂, 提高了材料的 活性比表面积，通过它们之间的协同作用整体提高催 化活性. 铜及其氧化物形成的复合催化剂具有高产氨 的选择性和活性, 为催化剂的设计提供了新的思路.

目前的研究主要集中于通过电化学性能测试了解 其催化反应的性能，但是具体的催化作用原理尚不清 晰. 为了深人了解电催化硝酸盐还原所涉及的多种电 荷转移，利用先进的原位表征技术，如X射线吸收光 谱、傅里叶变换红外光谱、拉曼光谱、X射线光电子 能谱和扫描透射电子显微镜, 探索和描述不同电催化 剂在其工作状态下的电子结构, 帮助我们了解反应物 分子在活性位点上的活化过程以及电化学反应中的结
构演变. 原位分析可以解析结构变化, 包括配位数、中 间体吸附或解吸引起的键长度, 以及电化学硝酸盐还 原过程中的应用电位. 这些信息对于了解反应途径和 催化剂中控制活性和选择性的关键结构因素是至关重 要的. 因此, 充分利用原位表征技术可以揭示电催化硝 酸盐还原反应的机理, 并指导高性能催化剂的设计.

理论计算可为解释反应机制的研究提供一些细节 和预测, 有助于对催化剂反应机制的解析. 然而, 理论 计算存在的问题是催化过程采用“静态”模型，而催化 剂在真实反应条件下是动态工作的, 因此, 将原位表征 技术与理论计算相结合才能了解结构与活性之间的关 系，并建立完整的理论体系. Wang 等人 ${ }^{[51]}$ 利用原位表 征技术和密度泛函理论相结合研究不同 $\mathrm{CuNi}$ 催化剂的 中间吸附与 $\mathrm{NO}_{3}{ }^{-}$还原活性之间的关系. 利用密度泛函 理论计算构建 $\mathrm{CuNi}$ 合金催化剂模型并进行验证，结果 显示 $\mathrm{d}$ 带中心费米能级上升，增强了中间的吸附能. 同 时，他们证明了 $\mathrm{d}$ 波段中心位置和诱导吸附特性与 $\mathrm{NO}_{3}^{-}$ 还原活性和产物选择性之间的关系，为合金催化剂的 设计指明了方向. 研究者利用反应机制的研究结果, 通 过机器的主动学习对潜在的电催化材料进行篮选, 再 通过实验测试对其进行验证，加快了高效催化剂的 发展.

将电化学硝酸盐还原推向商业化实际应用还面临 着很多挑战. 首先, 在放大反应体系中需要大面积的电 极，亟须克服传质所造成的影响; 其次，如何设计与搭 建合理高效的反应装置也成为高效硝酸盐还原的关键 所在; 最后, 若硝酸盐还原合成氨, 则涉及氨的分离收 集, 如何与现有的氨回收方法结合, 也是需要解决的问 题. 因此, 电化学硝酸盐还原技术的发展不仅要推动高 效催化剂的设计与制备，还需要提前布局研究产业化 所面临的科学问题.

\section{参考文献}

1 Kuypers M M M, Marchant H K, Kartal B. The microbial nitrogen-cycling network. Nat Rev Microbiol, 2018, 16: 263-276

2 Gruber N, Galloway J N. An Earth-system perspective of the global nitrogen cycle. Nature, 2008, 451: 293-296

$3 \mathrm{Fu}$ X, Zhao X, Hu X, et al. Alternative route for electrochemical ammonia synthesis by reduction of nitrate on copper nanosheets. Appl Mater Today, 2020, 19: 100620

4 Tugaoen H O N, Garcia-Segura S, Hristovski K, et al. Challenges in photocatalytic reduction of nitrate as a water treatment technology. Sci Total Environ, 2017, 599-600: 1524-1551

5 Rosca V, Duca M, de Groot M T, et al. Nitrogen cycle electrocatalysis. Chem Rev, 2009, 109: 2209-2244

6 Chen L, Zeng J, Li H, et al. Research advances in the soil nitrogen cycle under global precipitation pattern change (in Chinese). Acta Ecol Sini, 2020, 40: 7543-7551 [陈琳, 曾冀, 李华, 等. 全球降水格局变化下土壤氮循环研究进展. 生态学报, 2020, 40: 7543-7551] 
7 Samatya S, Kabay N, Yüksel Ü, et al. Removal of nitrate from aqueous solution by nitrate selective ion exchange resins. React Kinet Catal Lett, 2006, 66: 1206-1214

8 Comer B M, Fuentes P, Dimkpa C O, et al. Prospects and challenges for solar fertilizers. Joule, 2019, 3: 1578-1605

9 Gu Y W, Li X, Chen S, et al. Electrochemical method to remove nitrate in high-salt water (in Chinese). Chin Sci Bull, 2020, 65: 2880-2894 [厄⿱丆贝丨 薇, 李雪, 陈硕, 等. 高盐水中硝酸根的电化学去除方法. 科学通报, 2020, 65: 2880-2894]

10 Bhatnagar A, Sillanpää M. A review of emerging adsorbents for nitrate removal from water. Chem Eng J, 2011, 168: 493-504

11 Han L, Huang W, Yuan X, et al. Denitrification potential and influencing factors of the riparian zone soils in different watersheds, Taihu Basin. Water Air Soil Pollut, 2017, 228: 108

12 Alikhani M, Moghbeli M R. Ion-exchange polyHIPE type membrane for removing nitrate ions: Preparation, characterization, kinetics and adsorption studies. Chem Eng J, 2014, 239: 93-104

$13 \mathrm{Xu} \mathrm{D}$, Li Y, Yin L, et al. Electrochemical removal of nitrate in industrial wastewater. Front Environ Sci Eng, 2018, 12: 9

14 Huang W, Li M, Zhang B, et al. Influence of operating conditions on electrochemical reduction of nitrate in groundwater. Water Environ Res, 2013, 85: $224-231$

15 Hasnat M A, Rashed M A, Ben Aoun S, et al. Dissimilar catalytic trails of nitrate reduction on Cu-modified Pt surface immobilized on $\mathrm{H}^{+}$ conducting solid polymer. J Mol Catal A-Chem, 2014, 383-384: 243-248

16 Barrabés N, Sá J. Catalytic nitrate removal from water, past, present and future perspectives. Appl Catal B, 2011, 104: 1-5

17 Yin D, Liu Y, Song P, et al. In situ growth of copper/reduced graphene oxide on graphite surfaces for the electrocatalytic reduction of nitrate. Electrochim Acta, 2019, 324: 134846

18 Pennino M J, Compton J E, Leibowitz S G. Trends in drinking water nitrate violations across the united states. Environ Sci Technol, 2017, 51: $13450-13460$

19 Erisman J W, Sutton M A, Galloway J, et al. How a century of ammonia synthesis changed the world. Nat Geosci, 2008, 1: 636-639

20 Bandarenka A S, Koper M T M. Structural and electronic effects in heterogeneous electrocatalysis: Toward a rational design of electrocatalysts. J Catal, 2013, 308: 11-24

21 Wang Y, Zhou W, Jia R, et al. Unveiling the activity origin of a copper-based electrocatalyst for selective nitrate reduction to ammonia. Angew Chem Int Edit, 2020, 59: 5350-5354

22 Zhao Y, Kong F, Wang Z, et al. Role of membrane and compound properties in affecting the rejection of pharmaceuticals by different RO/NF membranes. Front Environ Sci Eng, 2017, 11: 20

$23 \mathrm{Lu} \mathrm{R}$, Chen W, Li W W, et al. Probing the redox process of p-benzoquinone in dimethyl sulphoxide by using fluorescence spectroelectrochemistry. Front Environ Sci Eng, 2017, 11: 14

24 Martínez J, Ortiz A, Ortiz I. State-of-the-art and perspectives of the catalytic and electrocatalytic reduction of aqueous nitrates. Appl Catal B, 2017, 207: $42-59$

25 Yu Y F. Electrocatalytic nitrogen cycling (in Chinese). Chin Chem Soc, 2019, 2: 30 [于一夫. 电催化氮循环. 中国化学, 2019, 2: 30]

26 Lan M N, Zhang Y L, Yao X Y, et al. Research progress of electrocatalytic reduction of nitrate for its removal from water (in Chinese). Appl Chem Ind, 2018, 47: 339-342 [兰孟娜, 张玉玲, 姚行艳, 等. 电催化还原水中 $\mathrm{NO}^{3-}$ 的研究进展. 应用化工, 2018, 47: 339-342]

27 Hamam A, Oukil D, Dib A, et al. Polypyrrole coated cellulosic substrate modified by copper oxide as electrode for nitrate electroreduction. Surf Rev Lett, 2015, 22: 1550065

28 Xiao Y, Zheng Y, Wu S, et al. Nitrogen recovery from wastewater using microbial fuel cells. Front Environ Sci Eng, 2014, 10: 185-191

29 Zeng Y, Priest C, Wang G, et al. Restoring the nitrogen cycle by electrochemical reduction of nitrate: Progress and prospects. Small Methods, 2020, 4: 2000672

30 van Langevelde P H, Katsounaros I, Koper M T M. Electrocatalytic nitrate reduction for sustainable ammonia production. Joule, 2021, 5: 290-294

31 Zhang R, Shuai D, Guy K A, et al. Elucidation of nitrate reduction mechanisms on a Pd-In bimetallic catalyst using isotope labeled nitrogen species. ChemCatChem, 2013, 5: 313-321

32 Tang C, Qiao S Z. How to explore ambient electrocatalytic nitrogen reduction reliably and insightfully. Chem Soc Rev, 2019, 48: 3166-3180

33 Sun $\mathrm{C}$, Li F, An H, et al. Facile electrochemical co-deposition of metal $(\mathrm{Cu}, \mathrm{Pd}, \mathrm{Pt}, \mathrm{Rh})$ nanoparticles on reduced graphene oxide for electrocatalytic reduction of nitrate/nitrite. Electrochim Acta, 2018, 269: 733-741

34 Wang P, Chu Y H. Research progress of copper-palladium electrocatalytic reduction of nitrate in water (in Chinese). Sichuan Chem Ind, 2020, 23: 14-17 [王鹏, 楚英豪. 铜钯电催化反硝化研究进展. 四川化工, 2020, 23: 14-17]

35 Zhang Y, Zhao Y, Chen Z, et al. Fe/Cu composite electrode prepared by electrodeposition and its excellent behavior in nitrate electrochemical removal. J Electrochem Soc, 2018, 165: 420-428

36 Filimonov E V, Shcherbakov A I. Catalytic effect of copper ions on nitrate reduction. Protect Met, 2004, 40: 280-285

37 Song Q, Li M, Wang L, et al. Mechanism and optimization of electrochemical system for simultaneous removal of nitrate and ammonia. J Hazard 
Mater, 2019, 363: 119-126

38 Yang J, Duca M, Schouten K J P, et al. Formation of volatile products during nitrate reduction on a Sn-modified Pt electrode in acid solution. J Electroanal Chem, 2011, 662: 87-92

39 Mattarozzi L, Cattarin S, Comisso N, et al. Electrodeposition of compact and porous Cu-Pd alloy layers and their application to nitrate reduction in alkali. Electrochim Acta, 2017, 230: 365-372

40 Dima G E, de Vooys A C A, Koper M T M. Electrocatalytic reduction of nitrate at low concentration on coinage and transition-metal electrodes in acid solutions. J Electroanal Chem, 2003, 554-555: 15-23

41 Long J, Chen S, Zhang Y, et al. Direct electrochemical ammonia synthesis from nitric oxide. Angew Chem Int Edit, 2020, 59: $9711-9718$

42 Shin H, Jung S, Bae S, et al. Nitrite reduction mechanism on a Pd surface. Environ Sci Technol, 2014, 48: 12768-12774

43 Liu R, Zhao H, Zhao X, et al. Defect sites in ultrathin Pd nanowires facilitate the highly efficient electrochemical hydrodechlorination of pollutants by $\mathrm{H}_{\text {ads. }}$. Environ Sci Technol, 2018, 52: 9992-10002

44 Anastasopoulos A, Hannah L, Hayden B E. High throughput optimisation of PdCu alloy electrocatalysts for the reduction of nitrate ions. J Catal, 2013, 305: 27-35

45 Reyter D, Chamoulaud G, Bélanger D, et al. Electrocatalytic reduction of nitrate on copper electrodes prepared by high-energy ball milling. J Electroanal Chem, 2006, 596: 13-24

$46 \mathrm{Wu}$ T, Kong X, Tong S, et al. Self-supported $\mathrm{Cu}$ nanosheets derived from $\mathrm{CuCl}-\mathrm{CuO}$ for highly efficient electrochemical degradation of $\mathrm{NO}^{3-}$. Appl Surf Sci, 2019, 489: 321-329

47 Wang X, Zhu M, Zeng G, et al. A three-dimensional Cu nanobelt cathode for highly efficient electrocatalytic nitrate reduction. Nanoscale, 2020, 12: $9385-9391$

48 Zhu T, Chen Q, Liao P, et al. Single-atom Cu catalysts for enhanced electrocatalytic nitrate reduction with significant alleviation of nitrite production. Small, 2020, 16: e2004526

49 Wang C, Liu Z, Hu T, et al. Metasequoia-like nanocrystal of iron-doped copper for efficient electrocatalytic nitrate reduction into ammonia in neutral media. ChemSusChem, 2021, 14: 1825-1829

50 Liu F. Fabrication and characterization of Cu/Ti bilayer nanoelectrode for electrochemical denitrification. Int J Electrochem Sci, 2016, 11: 83088322

51 Wang Y, Xu A, Wang Z, et al. Enhanced nitrate-to-ammonia activity on copper-nickel alloys via tuning of intermediate adsorption. J Am Chem Soc, 2020, 142: 5702-5708

52 Liu F, Li M, Wang $\mathrm{H}$, et al. Fabrication and characterization of a $\mathrm{Cu}-\mathrm{Zn}-\mathrm{TiO}_{2}$ nanotube array polymetallic nanoelectrode for electrochemically removing nitrate from groundwater. J Electrochem Soc, 2016, 163: E421-E427

53 Li M, Feng C, Zhang Z, et al. Simultaneous reduction of nitrate and oxidation of by-products using electrochemical method. J Hazard Mater, 2009, 171: 724-730

54 Zhang Y, Zhao Y, Chen Z, et al. Electrochemical reduction of nitrate via Cu/Ni composite cathode paired with Ir-Ru/Ti anode: High efficiency and $\mathrm{N}_{2}$ selectivity. Electrochim Acta, 2018, 291: 151-160

55 Weber B. Electro-catalytic reduction of aqueous nitrates using $\mathrm{Cu}-\mathrm{Sn}$ and $\mathrm{Cu}-\mathrm{Pd}$ cathodes. Int J Electrochem Sci, 2019, 14: 329-345

56 Mattarozzi L, Cattarin S, Comisso N, et al. Electrochemical reduction of nitrate and nitrite in alkaline media at CuNi alloy electrodes. Electrochim Acta, 2013, 89: 488-496

57 Mattarozzi L, Cattarin S, Comisso N, et al. Hydrogen evolution assisted electrodeposition of porous Cu-Ni alloy electrodes and their use for nitrate reduction in alkali. Electrochim Acta, 2014, 140: 337-344

58 Mattarozzi L, Cattarin S, Comisso N, et al. Electrodeposition of compact and porous $\mathrm{Cu}-\mathrm{Zn}$ alloy electrodes and their use in the cathodic reduction of nitrate. J Electrochem Soc, 2015, 162: D236-D241

59 Reyter D, Bélanger D, Roué L. Elaboration of Cu-Pd films by coelectrodeposition: Application to nitrate electroreduction. J Phys Chem C, 2009, 113: 290-297

60 Yang S. Electrochemical reduction of nitrate on different Cu-Zn oxide composite cathodes. Int J Electrochem Sci, 2017, 12: 4370-4383

61 Zhang Z, Xu Y, Shi W, et al. Electrochemical-catalytic reduction of nitrate over Pd-Cu/ $/ \mathrm{Al}_{2} \mathrm{O}_{3}$ catalyst in cathode chamber: Enhanced removal efficiency and $\mathrm{N}_{2}$ selectivity. Chem Eng J, 2016, 290: 201-208

$62 \mathrm{Li} \mathrm{W}$, Xiao C, Zhao Y, et al. Electrochemical reduction of high-concentrated nitrate using $\mathrm{Ti} / \mathrm{TiO}_{2}$ nanotube array anode and Fe cathode in dualchamber cell. Catal Lett, 2016, 146: 2585-2595

$63 \mathrm{Li}$ M, Feng $\mathrm{C}$, Zhang Z, et al. Efficient electrochemical reduction of nitrate to nitrogen using $\mathrm{Ti} / \mathrm{IrO}_{2}-\mathrm{Pt}$ anode and different cathodes. Electrochim Acta, 2009, 54: 4600-4606

64 Zhang L, Yin D, Zhai S, et al. Electrochemical behaviors and influence factors of copper and copper alloys cathode for electrocatalytic nitrate removal. Water Environ Res, 2019, 91: 1589-1599 
65 Gao W, Gao L, Meng J, et al. Preparation of a novel Cu-Sn-Bi cathode and performance on nitrate electroreduction. Water Sci Technol, 2019, 79: $198-206$

66 Couto A B, Santos L C D, Matsushima J T, et al. Hydrogen and oxygen plasma enhancement in the Cu electrodeposition and consolidation processes on BDD electrode applied to nitrate reduction. Appl Surf Sci, 2011, 257: 10141-10146

67 Chen M, Wang H, Zhao Y, et al. Achieving high-performance nitrate electrocatalysis with PdCu nanoparticles confined in nitrogen-doped carbon coralline. Nanoscale, 2018, 10: 19023-19030

68 Zhang Q, Ding L, Cui H, et al. Electrodeposition of Cu-Pd alloys onto electrophoretic deposited carbon nanotubes for nitrate electroreduction. Appl Surf Sci, 2014, 308: 113-120 


\title{
Progress on electrocatalytic reduction of nitrate on copper-based catalysts
}

\author{
Changhong Wang ${ }^{1 \dagger}$, Zhengyang Liu ${ }^{1,2 \dagger}$, Changming $\mathrm{Li}^{1,2^{*}} \&$ Chunxian Guo ${ }^{1 *}$ \\ ${ }^{1}$ School of Materials Science and Engineering, Suzhou University of Science and Technology, Suzhou 215009, China; \\ ${ }^{2}$ Institute of Advanced Cross-field Science, College of Life Sciences, Qingdao University, Qingdao 266071, China \\ $\dagger$ Equally contributed to this work \\ * Corresponding authors, E-mail: ecmli@swu.edu.cn; cxguo@usts.edu.cn
}

The rapid development of modern industry and agriculture has made serious nitrate pollution in groundwater, which has endangered human health and ecosystems. Electrocatalytic reduction is a new type of water treatment technology developed by combining electrochemistry and catalytic technology. By applying an electric current, an electrochemical reduction reaction of nitrate occurs at the cathode, thereby transforming nitrate into $\mathrm{N}_{2}$ or economically valuable $\mathrm{NH}_{3}$. Hence, to efficiently convert nitrate in water is alluring from the perspective of environmental protection and energy saving. The key of nitrate electrochemical reduction technology is the cathode material. The optimization of cathode materials is vital to push the development and application of electrocatalytic reduction of nitrate. Good catalytic materials require higher catalytic activity and product selectivity, as well as stability and corrosion resistance in sewage. Copperbased materials have become a research hotspot due to their intrinsic catalytic activity. Currently, researchers have mainly explored metallic copper, single-atom copper, copper alloy and copper based composites. However, it is still a lack of related review to discuss the structure-activity relationship between the structure of copper-based materials and the electrocatalytic nitrate reduction performance.

This paper mainly reviews the research progress of electrocatalytic nitrate reduction of copper-based material catalyst, and analyzes the relationship between structure and its selectivity and catalytic activity from the perspective of single matter copper, single atomic copper, copper alloy and copper-based composite materials, respectively. First, the surface defects of the exposed single copper were regulated by changing its surface morphology and then optimized the electrocatalytic nitrate reduction. Because of single copper corrosion resistance and poor catalytic stability, it is not easy to use for nitrate reduction. Secondly, single atomic copper electrode materials exhibit high activity and selectivity in the catalytic reaction. However, how to achieve the high load capacity of a single atom catalyst is an urgent problem for researchers to break through. Then, for the copper alloy, the stability of the catalyst is greatly improved, and because of the synergistic electron effect between metals, the multimetal electrode performance is stable and has corrosion resistance, and the catalytic activity is higher than the ordinary single metals. However, the copper alloy, especially the copper alloy mixed with precious metal, increases the corresponding cost, which is not conducive to the industrial promotion of a large area. Finally, copper and non-metal material carriers form a composite catalyst, which can combine the advantages of low cost, high mechanical strength, strong tunability and good durability of electronic structure. The uniformly dispersed copper nanocatalysts improve the active ratio and surface area of the material, and improve the catalytic activity through the overall cooperation between them. The composite catalyst of copper and its oxides also provides new ideas for the design of the selectivity and activity of high yield ammonia. The future development direction of copper-based materials and the challenges faced by actual industrial applications are discussed.

electrocatalysis, nitrate reduction, copper-based catalysts, structure-activity relationship doi: 10.1360/TB-2021-0333 